\title{
La regeneración de la laguna de Venecia a través de la interpretación de sus paisajes culturales
}

\author{
Gemma Milà Cartañá \\ gemma.mila.ca@gmail.com
}

\begin{abstract}
Resumen. La laguna Veneta consta de aguas poco profundas, marismas, islas y una red de canales. Estos elementos fueron integrados en la cultura local desde el primer asentamiento romano. El planeamiento y posición de islas artificiales, permitiendo el acceso y renovación del agua según las mareas, o la creación de una red de huertos salados mediante el sistema de canales existente, favorecedor de la vegetación halófila, son ejemplos de la complementariedad entre este territorio característico y su cultura particular.

La constelación formada por territorios militares abandonados junto a los ecosistemas terrestres y palustres que los han reconquistado son una ocasión extraordinaria para su recuperación y valorización social, económica, histórica y ecológica. Aun formando parte de un sistema global de defensa lagunar, las micro-realidades de estas entidades son diversas. La experimentación de nuevos métodos y usos contemporáneos se adhiere a las dinámicas de mutación características de estas zonas a lo largo de la historia. La regeneración global de la laguna a través de esas acciones específicas, contextualizadas en la totalidad del territorio, puede ser una oportunidad para la revitalización de la complejidad económica y cultural de la región, actualmente reducida mayoritariamente al turismo de masas.
\end{abstract}

El reconocimiento y la percepción del sistema defensivo formado por arquitecturas arquetípicas y ecosistemas preservados proporcionan un enfoque multidisciplinar desde la matriz biofísica hasta las prácticas humanas en estos espacios tan singulares. Tal interpretación de los paisajes culturales de la laguna puede ser un medio para revelar y acompañar las dinámicas del territorio lagunar.

Palabras clave: regeneración de la laguna de Venecia, paisajes culturales, simbiosis entre vegetación y vestigios históricos, zonas militares abandonadas, vía verde y de patrimonio, Lido de Venecia.

Abstract. The Venetian lagoon is composed of shallow waters, marshes and a canal net among islands. These elements were integrated in the local culture since the first roman settlement. This particular territory and its culture are extremely interrelated. We have found many examples, as the planning and placement of the artificial islands allowing the access and renovation of water when tides, or the creation of a salty agriculture system that supports the development of halophytic vegetation. Cultural landscape is an interesting entity to explore in this territory. The balance of this environment is very weak due to opposite forces, like the river sedimentation confronting the sea erosion. The culture is also paradoxical with regard to the local arts and crafts specific of each island contrasting with the economy interests of the international harbour.

The constellation formed by the military abandoned territories and the land and water ecosystems that had regained them are an extraordinary opportunity to recover and revalue the social, economic, historical and ecological lagoon dynamics. Even though these territories belong to a global defence system, each one has a very different micro-reality, and the interventions become challenging at this small scale. The experimentation of new methods and contemporary uses fits in with the changes of this territory along the history. When the local intervention spreads along the territory can reach the opportunity to regenerate the lagoon that nowadays is degradated because of the global tourism.

The awareness and perception of the defensive system formed by archetypal architectures and preserved ecosystems give a multidisciplinar point of view of the territory from the biophysic matrix to human practices. This interpretation of the cultural landscapes can be a tool to reveal and foster the dynamics of the venetian territory.

Keywords. Venice lagoon regeneration, cultural landscapes, simbiosis among vegetation and vacant historical territories, vacant military zones, green and heritage corridor, Lido of Venice. 


\section{Introducción}

Esta investigación nació de unos talleres internacionales sobre la intervención en paisajes históricos, en el marco universitario del Instituto Universitario de Arquitectrua de Venecia, y de la Universidad Saint Luc de Bruselas. Dos años más tarde estas reflexiones sobre la isla de Venecia y sus entornos se desplegaron para concebir un proyecto de fin de carrera sobre la regeneración de la laguna de Venecia.

A través del aprendizaje y utilización de Sistemas de Información Geográfica y el ejercicio de superposición de diferentes estratos del territorio que pudieran crear relaciones "layercake" (McHarg, 2000), llegamos a la conclusión que la isla del Lido era una de las zonas de la laguna de Venecia más deterioradas a causa de una multitud de factores que habían reducido su complejidad y alterado su mosaico territorial característico a lo largo de la historia.

La intervención y proyecto en la isla del Lido se desarrolló en torno a la cuestión de los paisajes culturales. La búsqueda del sentido de este concepto en un contexto específico es fundamental para la materialización de esta investigación.

La interacción con distintos actores del territorio ha sido esencial para la comprensión del mismo así como la colaboración de actores externos de disciplinas muy distintas. Igualmente lo ha sido el estudio de las temáticas abordadas a través de teorías globales así como la comparación de referencias en otros contextos.

La aproximación multidisciplinar ha sido la base de esta investigación así como el estudio del territorio a través de experiencias distintas sobre este lugar excepcional.

\section{Contexto geomorfológico y perspectiva histórica de la laguna de Venecia}

El mar Adriático está cerrado en su extremo septentrional por el golfo de Venecia. En él, la confluencia de las aguas que descienden de importantes cordilleras ha creado un sistema de lagunas de grandes dimensiones, que tienen características diversas según cómo sean los extensos territorios que ocupan. Así, la magnitud, en superficie y altura, del sistema orográfico situado al noreste de esta región ha generado, por erosión, transporte y sedimentación, la extensa llanura aluvial del Veneto, dotada de una red hidrográfica y lagunar densa. Los ríos originados en la región Veneta y la Trentina por las nieves de los Dolomitas dan lugar a esa gran llanura inundada por todo el territorio y, al llegar al mar, la interacción entre los abundantes caudales de agua continental y el sistema marino ha modelado la laguna de Venecia.

El gran aporte de sedimentos por parte de los cursos fluviales más importantes era capaz de colmatar dicha laguna. Por este motivo, a lo largo de la historia, las comunidades que habitaban en ella planificaron la desviación de estos ríos y perpetuaron el sistema lagunar. Por otro lado, las ricas llanuras agrícolas que envuelven este golfo han hecho que esas tierras fueran productivas y generaran sociedades desarrolladas que rivalizaban entre ellas. El mar ha sido, por lo tanto, objeto de control y vigilancia durante todas las épocas. 


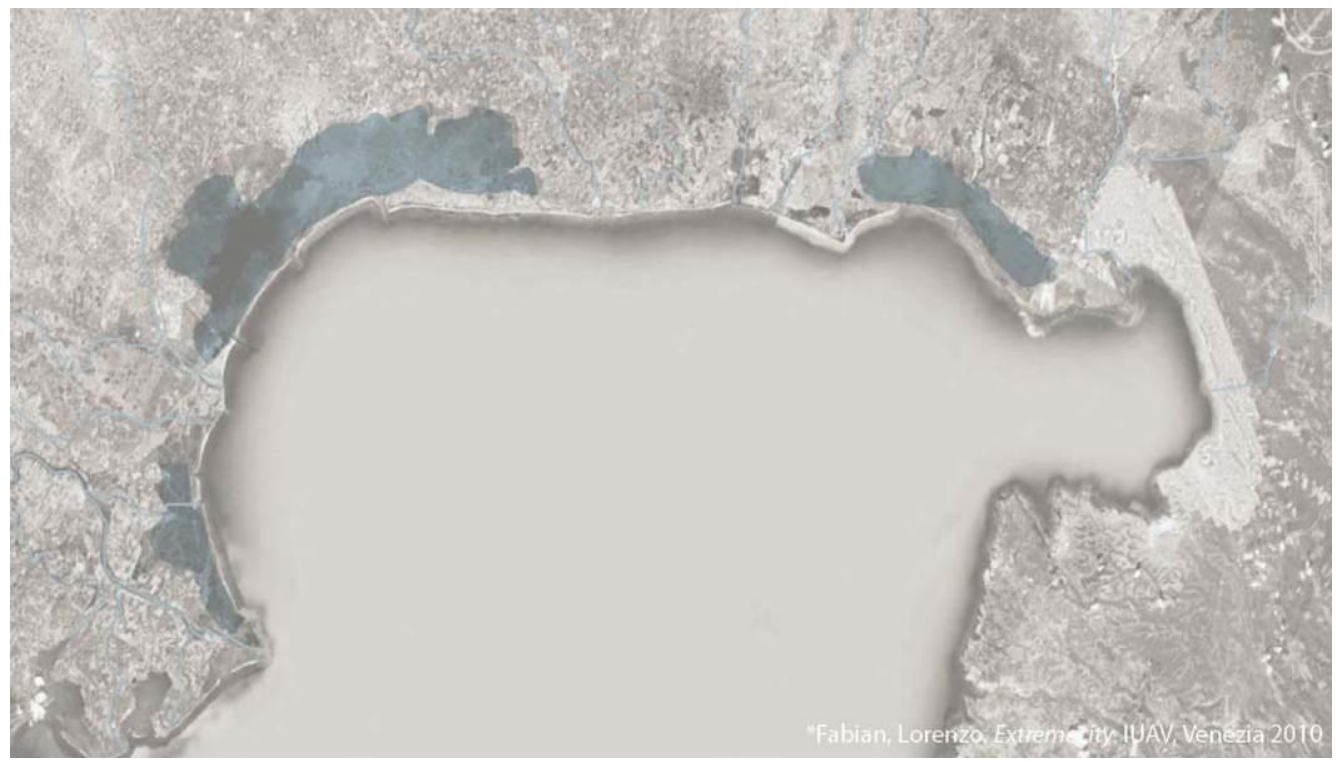

Fig. 1. Sistema lagunar del golfo del mar Adriático.

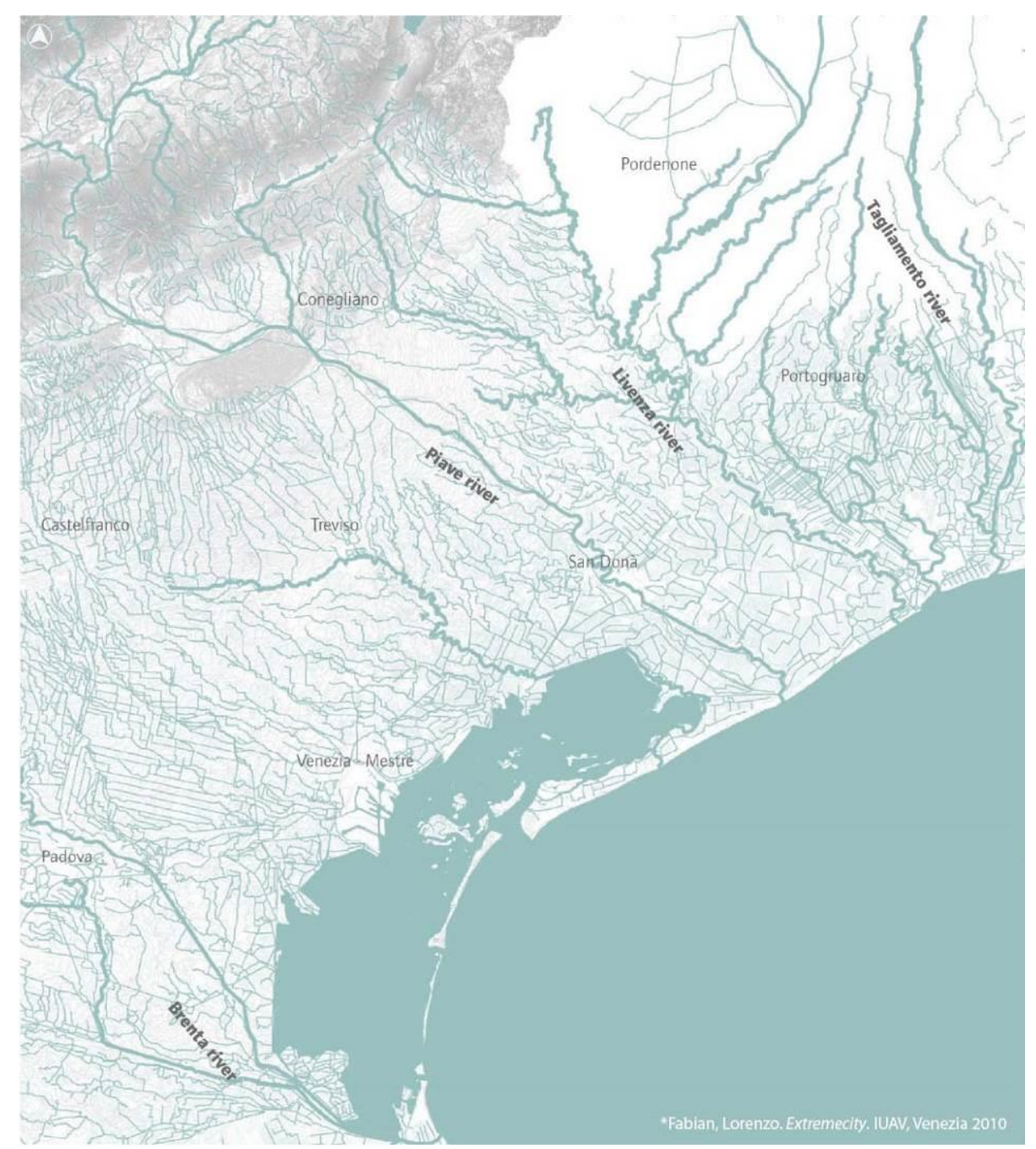

Fig. 2. El sistema orográfico de los Dolomitas, la llanura del Veneto y la laguna de Venecia. 
Lido y Pellestrina representan las islas litorales de la laguna que compartían un perfil territorial característico y forman parte de la misma municipalidad. Tanto la morfología de las islas como su población han sufrido muchos cambios durante las diferentes épocas de la historia y sobretodo actualmente.

La posición de las islas litorales en la laguna es particular ya que su tamaño y forma son completamente distintos del resto del sistema: se trata de dos islas de más de diez kilómetros de costa pero de una media de quinientos metros de ancho. La distancia entre la isla de Venecia y cada isla litoral es muy diferente y, en consecuencia, la influencia de aquella es muy distinta.

Estas islas tenían una relación con la laguna muy estrecha y se protegían del mar Adriático, que siempre fue un enemigo para estas tierras tan frágiles debido a la erosión. La configuración artificial de las entradas a la laguna desde el mar (llamadas «bocca») ha sesgado la continuidad litoral y lagunar.

La industria del turismo cambió la manera tradicional de obtener recursos de la laguna de Venecia, obteniéndolos de la costa Adriática a partir de entonces. La obertura de «Des Bains» (primer gran hotel de la costa mediterránea europea en el siglo XVIII) aumentó la popularidad de las playas de las islas litorales, con la consecuencia de la construcción exponencial de apartamentos y hoteles en los años setenta y ochenta del siglo XX. Así, las prácticas del turismo de costa se vieron reemplazadas por el turismo de masas que convirtió las islas del litoral, sobretodo la isla del Lido di Venezia (la más cercana a Venecia) en una opción mucho más económica para las pernoctaciones en los viajes destinados únicamente a visitar la isla de Venecia.

\section{Situación actual de la isla del Lido de Venecia y sus paisajes culturales}

El Lido es una isla que ha vivido muchos cambios en diferentes escalas y estratos del territorio. Su situación tan particular y a la vez frágil -entre la laguna y el mar- determina las fuentes de recursos naturales, sociales y económicos de sus habitantes.

La diversidad de la isla (sobre todo la biodiversidad) se ha visto reducida por el incremento de la presión humana sobre el territorio, causada por la introducción de factores externos que produjeron la transformación morfológica y del mosaico de la isla. Por ejemplo, el crecimiento y movimiento de la población, la configuración de las entradas de embarcaciones desde el mar hacia la laguna, la diversificación artificial del mosaico territorial de la isla debido a las distintas recalificaciones y divisiones de las zonas características, así como la desconexión de la laguna como fuente de recursos, causada por la subordinación al turismo de masas de la isla de Venecia.

Pero para entender las dinámicas de transformación actuales hay que analizar también las dinámicas geomorfológicas de la isla: el Lido se estrecha a causa de la erosión del mar en su parte central, en donde la corriente marina reduce las playas; en cambio, en sus extremos, en donde se construyeron unos diques para asegurar la dimensión de las entradas a la laguna, la aportación de sedimentos por parte del mar ensanchó esos terrenos. Así, la controversia entre las dinámicas de sedimentación y erosión desequilibraron la continuidad del sistema dunar, que fue disminuyendo en el centro y creciendo en los extremos de la isla. 


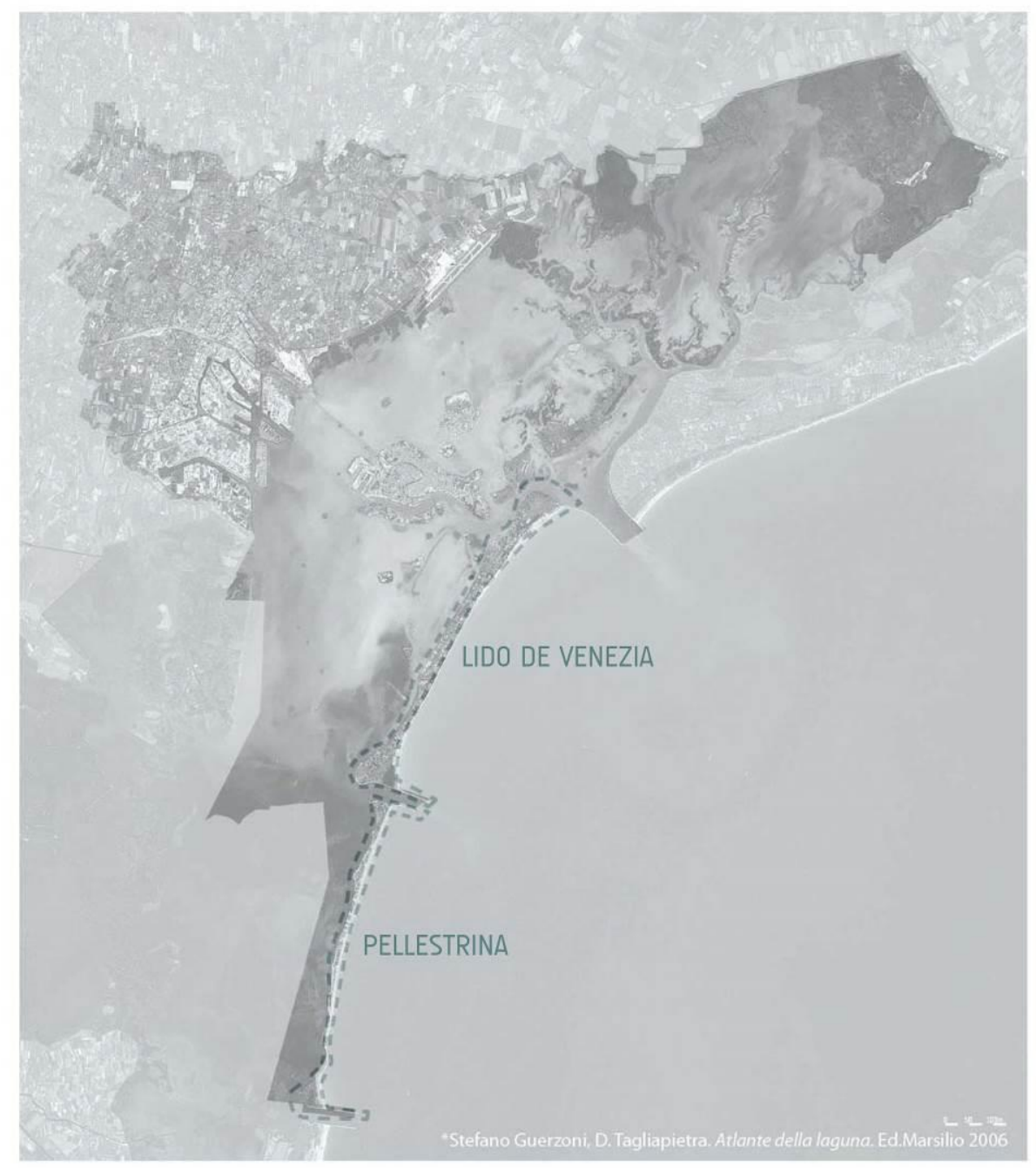

Fig. 3. Region del Veneto : las islas litorales del Lido de Venecia y Pellestrina.

Otro indicador de los cambios y desequilibrios actuales de la isla es el movimiento de la población y sus asentamientos: La isla tenía un solo poblado llamado Malamocco. Este fue capital de la República Sereníssima de Venecia en sus principios (siglo XV). El resto de la isla estaba constituido por un importante sistema dunar: un gran bosque litoral cubría una gran parte de la isla. Este paisaje era transformado en puntos donde se localizaban acequias -«ghebbi»- que drenaban el agua salobre de la laguna hacia los campos agrícolas de la isla conocidos como «orti salatì. En esa época dos fuertes coronaban los extremos de la isla («forte Alberoni» y «San Nicoló») y con sus grandes fosas y murallas puntiagudas vigilaban la entrada y salida de embarcaciones en la laguna de Venecia. Cuando en épocas posteriores se abandonaron y destruyeron, la vegetación aprovechó el nuevo relieve para instalarse. $\mathrm{Al}$ mismo tiempo se empezó a manipular el relieve de las islas con la creación de las entradas a la laguna desde el mar y consiguiente sedimentación en los dos extremos de la isla: éstos adquirieron una gran importancia ecológica gracias al intenso desarrollo del ecosistema dunar. Aun así, durante el esplendor de la República Sereníssima, la construcción de barcos era una de las prácticas más importantes. Las naves se construían en madera que se cortaba de los bosques dunares, que desaparecieron poco a poco. 
Estas zonas desforestadas se edificaron a causa de las prácticas de veraneo de las clases altas. En el siglo XVIII se construyeron: un núcleo de segundas residencias -villas- cercano al fuerte San Nicoló, que fue abandonado; un complejo -balneario de élite- dedicado al turismo; y una trama de casas nobles unifamiliares entorno a la avenida Santa Maria Elisabetta, que es una avenida que conecta la laguna y las playas a la misma latitud que la isla de Venecia. La morfología dunar fue destruida pero el tejido era generoso en jardines. Como hemos dicho, el fenómeno del turismo de masas llegó a la isla del Lido en los años ochenta, obligando a construir y densificar este núcleo. Las parcelas de las villas fueron segmentadas para densificar el espacio. Un tejido de inmuebles de tres y cuatro plantas ocupó todo el territorio norte de la isla.

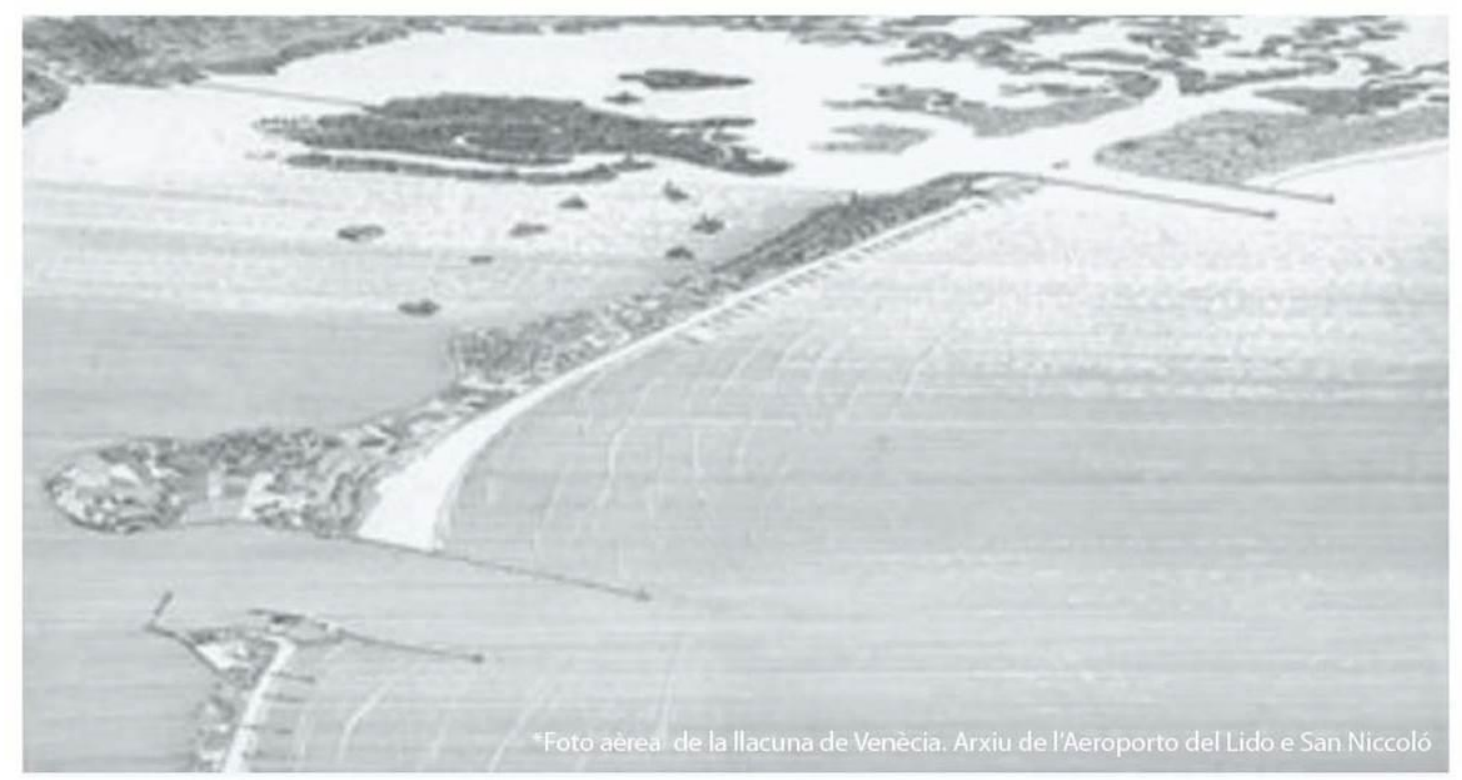

Fig. 4. La isla del Lido de Venecia.

La vegetación autóctona de la isla del Lido, como pueden ser las comunidades psammófilas (Ammopbila arenaria,...) y halófilas (Tamarix sp.,...), ha sido prácticamente extinguida, quedando sólo algunos especímenes aislados. Aun así, es posible entender el territorio y reconstruir el ecotono entre las dos zonas litorales: entre laguna y mar. Actualmente, las comunidades del litoral marítimo están mucho más representadas en los extremos norte y sur de la isla a consecuencia de la sedimentación del mar causada por la construcción de diques perpendiculares a la isla para asegurar las entradas navales de la laguna. En cambio, el crecimiento de las comunidades del litoral lagunar es mucho más limitado, ya que un canal artificial dragado periódicamente delimita el perímetro interior de la isla e impide la transición gradual del medio acuático al terrestre. En los intersticios que ofrece el interior de la isla crecen comunidades distintas con un grado artificial elevado (cañizales, arboledas, zarzales, ...). Por lo tanto, el mosaico territorial está muy dividido y es muy distinto según la zona de la isla: la fracturación del paisaje impide una lectura global del territorio. 


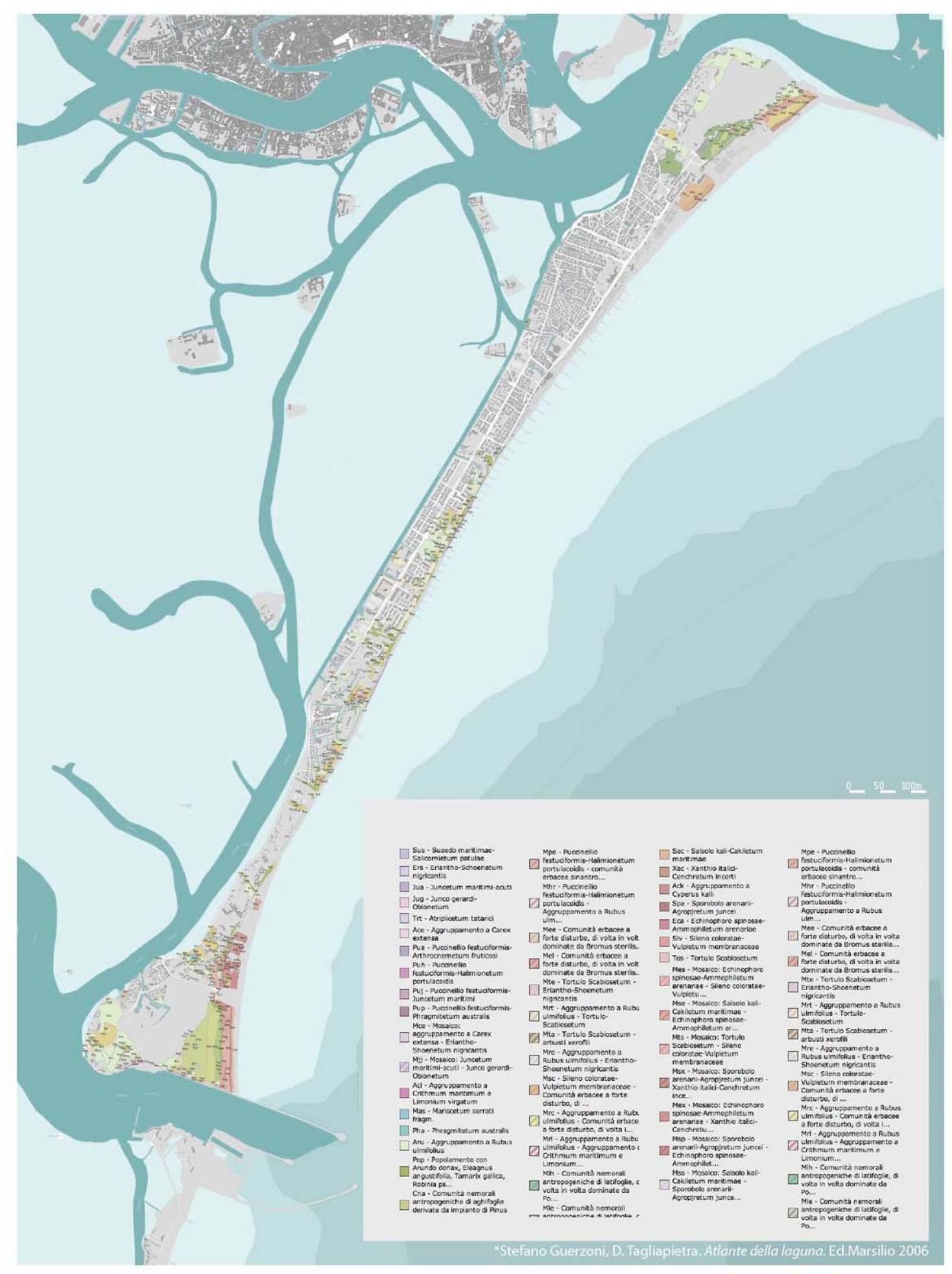

Fig. 5. Comunidades vegetales de la isla del Lido. 
Las edificaciones de defensa de la laguna contra los ataques navales y posteriormente aéreos son una estrategia militar desarrollada desde el siglo XV y se sitúan a lo largo de toda la costa adriática de la laguna de Venecia con un ritmo constante.

Las primeras construcciones fueron el fuerte de San Nicoló en el norte de la isla del Lido de Venecia. Junto con el fuerte de San Andrea (al lado de la isla de La Vignole) constituían un sistema defensivo frente al acceso septentrional a la laguna. Desde la República de la Sereníssima (siglo XV) la protección naval militar se concibe a través de una estrategia de fuertes situados en los márgenes de la laguna. Durante el imperio Austrohúngaro (siglo XIX), estos fuertes se rehabilitaron y se añadieron otros según las estrategias militares del momento (nuevas tecnologías de guerra). En la primera guerra mundial se aprovecharon estos fuertes y se añadieron baterías de cañones que también se colocaron en otros puntos según las estrategias tecnológicas del momento. En la segunda guerra mundial, temiendo un desembarco de los aliados por el golfo del mar Adriático, se construyeron una serie de bunkers de artillería, municiones, antiaéreos y torres de comunicación alrededor de las antiguas fortalezas y en nuevos puntos estratégicos que daban continuidad a este muro protector de bunkers: una estrategia militar similar al «Atlantikwall».

Actualmente estos edificios, que reflejan distintas épocas de la historia de la región, están abandonados y escondidos entre la vegetación, anulando su percepción y testimonio de la historia.

Las edificaciones militares comportan connotaciones y recuerdos desagradables para las personas que conviven con estas construcciones. El hecho de que estos edificios sean concebidos con un fin puramente técnico y específico hace difícil la apropiación contemporánea, integrándolos a la cotidianeidad de las personas. Estas edificaciones son objeto de abandono y repudio ${ }^{1}$. Estas dinámicas han abducido este patrimonio al abandono, demolición o delegación de terrenos a propiedades privadas no comprometidas.

1 Teoría concebida por Paul Virilio (1994): "Defensive architecture is therefore instrumental, existing less in itself than with a view to "doing" something: waiting, watching, (...) To live in such a place is not so much to "dwell" there as it is to "take it on" for an act for wich the casemate is the instrument." 


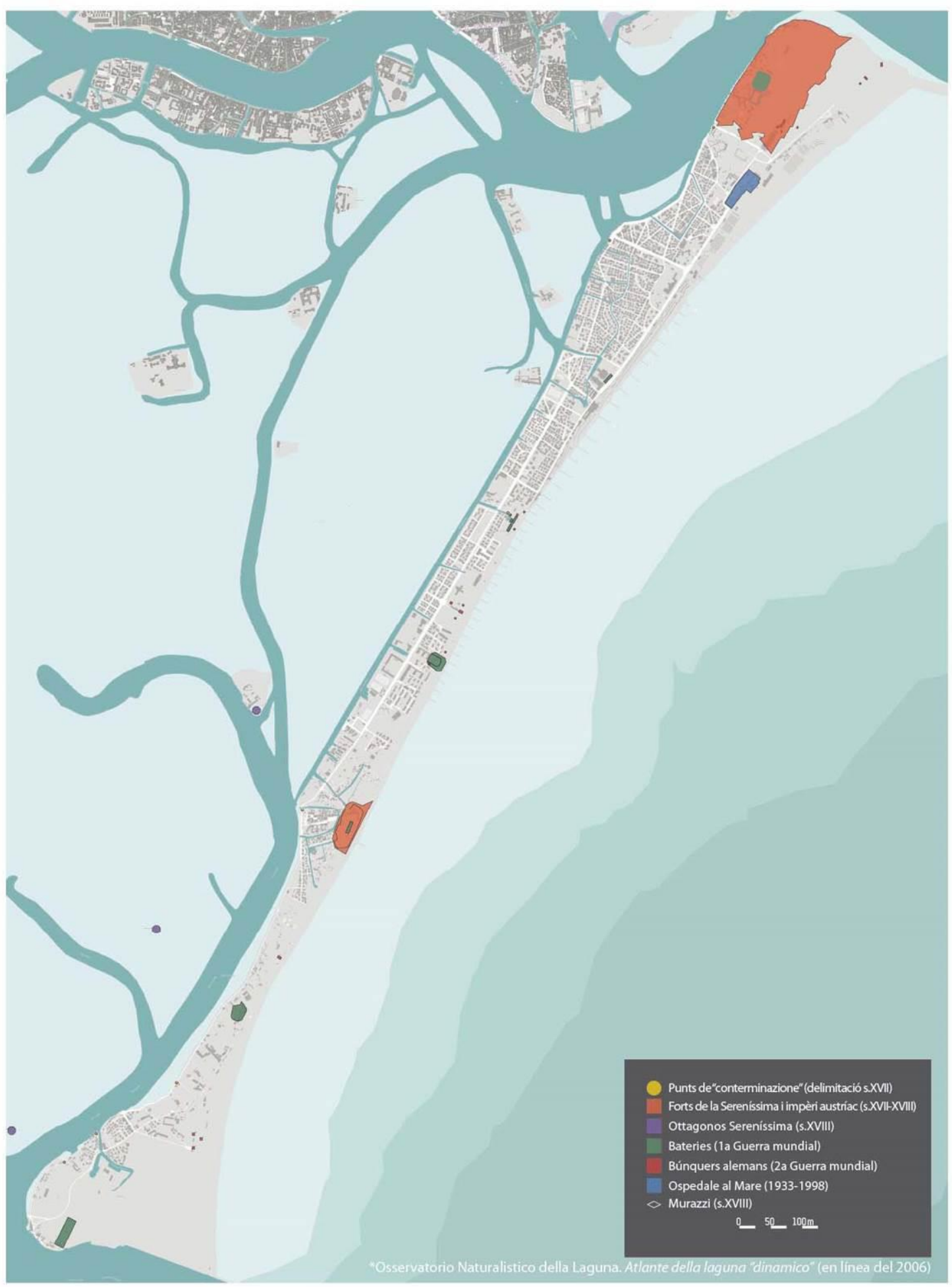

Fig. 6. Patrimonio histórico militar. Leyenda: Puntos de conterminazione, Fuertes de la Sereníssima, Baterias de la primera guerra mundial, Bunkers alemanes de la segunda guerra mundial, Ospedale al mare, Murazzi. 


\section{Propuesta de planificación del territorio a través de la relación entre patrimonio natural e histórico}

Como hemos indicado, la isla del Lido ha vivido muchas transformaciones. La proximidad de Venecia ha comportado una presión muy fuerte sobre el Lido alterando su "metabolismo". La subordinación a la isla de Venecia ha hecho que la ordenación del territorio del Lido se desequilibre para cubrir las necesidades procuradas por la economía, sobretodo la industria del turismo. El mosaico territorial es muy distinto según el tramo de la isla y su relativa distancia a la isla de Venecia creando una discontinuidad del paisajes muy radical.
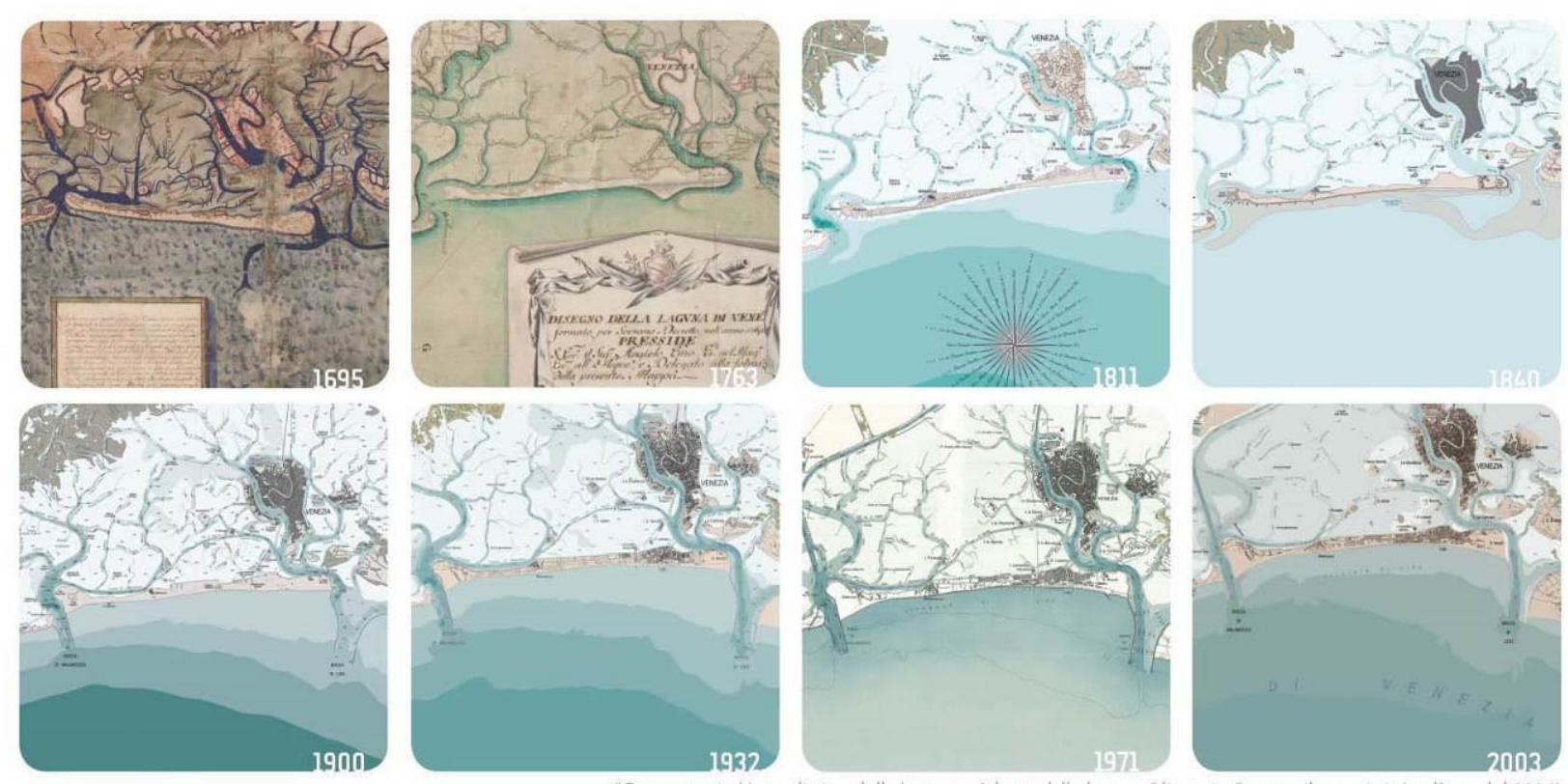

Osservatorio Naturalistico della Laguna. Atlante della laguna "dinamico". www.silvenezia.it (en línea del 2006)

Fig. 7. Cartografía histórica de la isla del Lido de Venecia (Guerzoni y Tagliapietra, 2006).

El cambio de centralidad (de Malamocco a Lido) y el crecimiento exponencial entre los años setenta y noventa del siglo XX, ha hecho que la mitad septentrional de la isla se edificara con la consiguiente privatización y explotación de las playas, degradando la vegetación y transformando la morfología autóctona (el sistema dunar). La parte meridional de la isla (Malamocco) es mucho más agrícola ya que toda la línea del litoral marítimo está cerrada por un gran muro de protección contra la erosión. Aun así, la presión del turismo en la parte septentrional de la isla influye en la parte sur ya que se están empezando operaciones inmobiliarias en los campos agrícolas abandonados a causa del éxodo rural hacia la parte septentrional para trabajar en el sector terciario. 


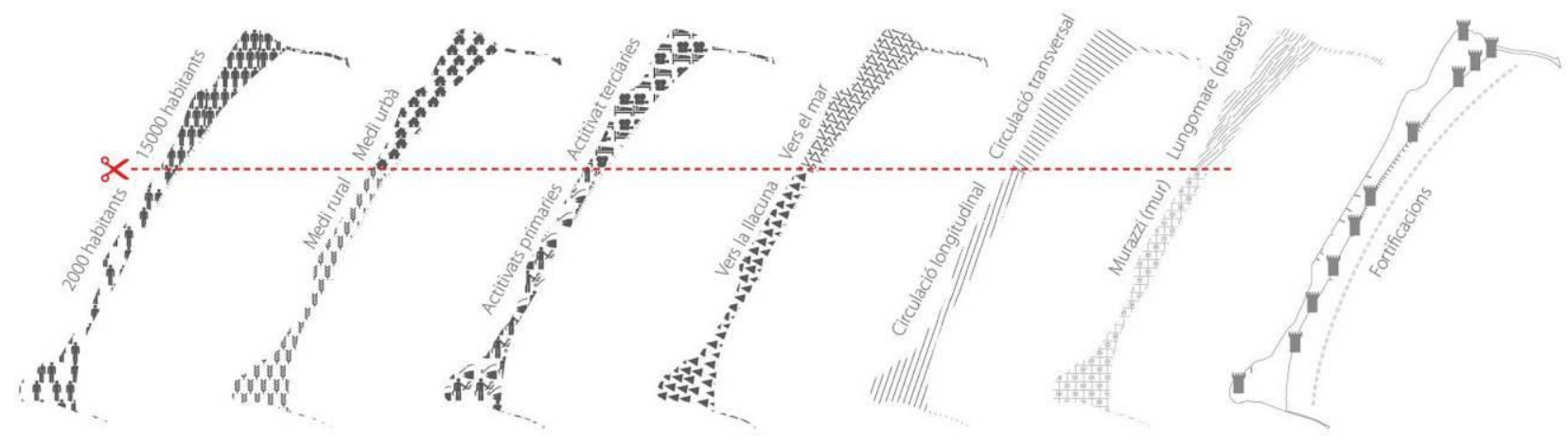

Fig. 8. Dicotomías del territorio del Lido. De izquierda a derecha: 1. Número de habitantes, 2. Medio: natural/urbano, 3. Tipo de actividades: primarias/terciarias, 4. Fachada hacia: laguna/mar,5. Circulación: longitudinal/transversal, 6. Tipo de litoral marítimo:

Murazzi/Lungomare, 7. Continuidad de las edificaciones militares a lo largo de la isla.

Esta dicotomía del paisaje entra en conflicto en la parte central de la isla donde el límite de los municipios del Lido y Malamocco es difuso. El límite entre las dos poblaciones está compuesto de terrenos intersticiales; una parte importante de estos terrenos son zonas militares abandonadas con vestigios de bunkers y baterías de la segunda guerra mundial.

La localización de estas zonas militares corresponde a las zonas de comunidades vegetales importantes. Esta cohabitación se podría explicar como consecuencia de la falta de interés por los espacios naturales y arqueológicos debida al fenómeno de la urbanización y al turismo de masas. Las comunidades vegetales y el patrimonio militar se encuentran actualmente en una misma situación de deterioro pasivo en puntos concretos del territorio. Por lo tanto, esta situación podría calificarse de simbiótica ya que las construcciones militares protegen las especies vegetales e inversamente, sobre todo porque las comunidades exuberantes de la periferia de la zona actúan como pseudoecotono y protegen les edificios del acceso humano indebido. Podríamos denominar "entidades expectantes" a estas entidades del territorio, porque se encuentran en un período de degradación pasivo y latente a la espera de una intervención de regeneración. Estas entidades se extienden a lo largo de toda la isla y atraviesan los diferentes ambientes del mosaico territorial. Esta regularidad nos hace intuir una posible intervención para regenerar el territorio y crear una continuidad del paisaje.

Para ello nos fijaremos en que el análisis de los paisajes culturales de la isla distingue cuatro ámbitos de norte a sur. Cada ámbito comparte un conjunto de características biofísicas, patrimoniales y geográficas particulares. En cada ámbito podemos hacer una propuesta de regeneración de las zonas expectantes de carácter distinto pero siguiendo el mismo objetivo: la continuidad y creación de una vía verde y de patrimonio. Estas intervenciones en las realidades puntuales deberían reflejar una coherencia global de los paisajes culturales de la isla del Lido. Resumamos, entonces, cuáles son nuestras propuestas para los cuatro ámbitos del Lido. 


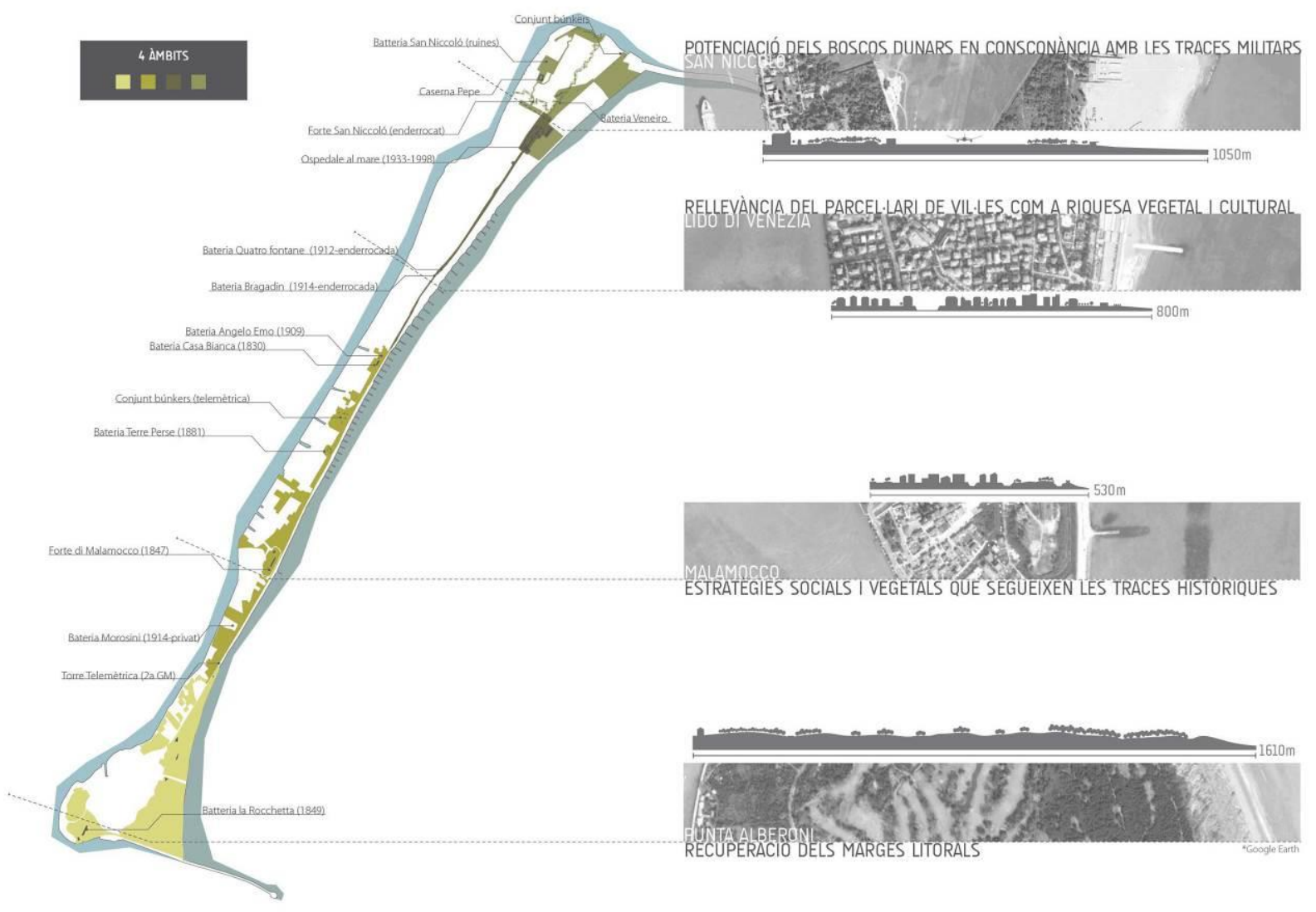

Fig. 9. Cuatro ámbitos. De norte a sur, ámbito y intervención: Ámbito de San Niccoló: Potenciación de los bosques dunares en consonancia con las trazas militares; Ámbito del Lido de Venécia: Revalorizar el parcelario de las villas como una riqueza vegetal y cultural; Ámbito de Malamocco: Estrategias sociales y vegetales que sigan las trazas históricas; Ambito de Punta Alberoni: Recuperación de los bordes litorales.

- El ámbito norte está constituido por el antiguo fuerte de San Nicoló, el gran fuerte que controlaba la entrada a la laguna por el norte del Lido. Esta gran fortificación fue destruida y rehabilitada por el imperio Austrohúngaro y finalmente, en las últimas grandes operaciones militares de la historia, se realizó una serie de construcciones militares entorno al fuerte frente al mar Adriático (baterías antiaéreas italianas, sistema de bunkers alemanes, etc.). Actualmente este territorio tiene unas características comunes gracias a las grandes «arboledas» interiores y a las construcciones militares regularmente diseminadas en el entorno del actual aeródromo construido sobre la llanura central del fuerte, donde se encuentran dichas intervenciones militares más recientes.

Este territorio tiene pues un marco histórico muy interesante dominado por las intervenciones militares a gran escala. Además, este ámbito presenta un valor naturalístico importante ya que la sedimentación en los diques de la entrada a la laguna ha ensanchado el medio pudiendo regenerarse el sistema de dunas.

El descubrimiento, reconocimiento y conexión de los edificios militares expandidos por el territorio podría ser el motor de la regeneración de las comunidades vegetales (sobre todo las importantes arboledas de las dunas secundarias) y los trazados de paseo existentes ahora inconexos y descuidados. La creación de un paseo a través de la rehabilitación de las pistas existentes sería un 
ejercicio interesante de observación y de experiencia. Los usuarios podrían sentir este territorio más cercano gracias a sus vivencias y este comportamiento podría inducir un respeto y cuidado de éste. ${ }^{2}$

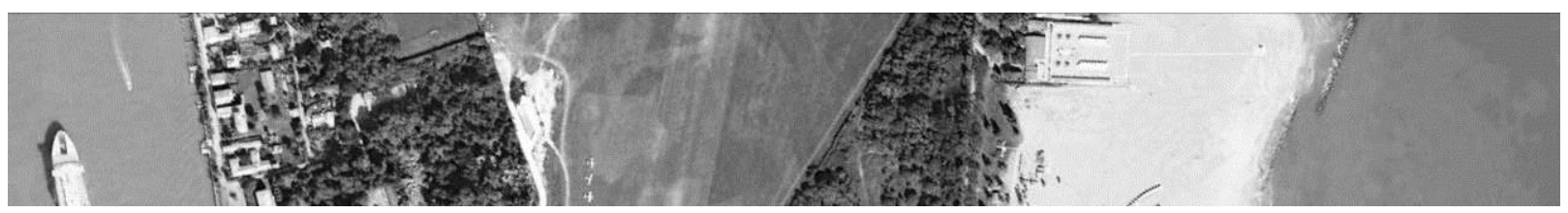

Fig. 10. Foto aérea del ámbito de San Niccoló

- El ámbito del municipio del Lido es el más urbano. La privatización de las playas ha hecho que el paseo marítimo pierda valor y se degrade, obteniendo un estatus de carretera no apta para los peatones. El desplazamiento en bicicleta es común entre los habitantes del Lido. Hay proyectos de recalificación del espacio público para conceder más importancia a este medio de transporte. Dentro del pueblo se encuentran las villas antiguas de finales del siglo XIX envueltas de jardines amanerados que refrescan el ambiente cálido de la urbe. Aún así, las construcciones más modernas que se intercalan entre las villas estan muy degradadas y entorpecen la lectura de los recorridos históricos. La red de calles está degenerada y llena de dificultades para el peatón.

La regeneración y adaptación del pueblo del Lido se podría enfocar a través de la valorización del itinerario, ya existente, para visitar estas obras arquitectónicas. Esta estrategia serviría para movilizar a los agentes públicos (la red de calles) asi como a los privados (la regeneración de fachadas y espacios abiertos interiores de las parcelas privadas).

Siguiendo las indicaciones del plan regulador del Lido de Venecia, las actividades hoteleras del paseo maritimo y playas tendrian que estar mucho más relacionadas con actividades y curas balnearias como lo estuvieron históricamente las playas del Lido. Esta aproximación al bienestar relacionado con el mar debería propulsar el cambio del tipo de mantenimiento de las playas (actualmente tabula rasa de las dinámicas litorales) para adaptarse a las dinámicas naturales (sistema dunar). De la misma manera, todas estas actividades deberían promover la regeneración del paseo maritimo, haciéndolo más apto para peatones y bicicletas.

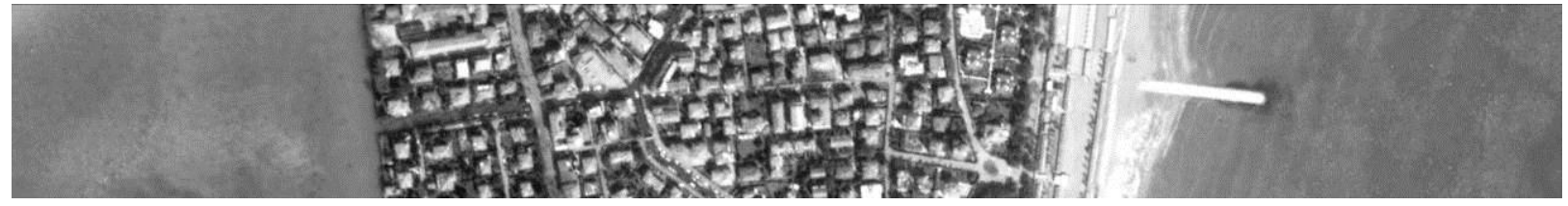

Fig. 11. Foto aérea del ámbito del Lido

2 Teresa Galí-Izard (2006) "Un simple paseo por el campo se transformó en un interesante ejercicio de observación. [...] Hacían fotografías desde el mismo punto de vista, dibujaban y anotaban todo aquello que les llamaba la atención de forma regular y sistemática. [...] Al cabo de un tiempo, el conocimiento y la vivencia que habían adquirido hizo que sintieran el lugar como propio". 
- El ámbito del Murazzi empieza al final del municipio del Lido y termina en la punta Alberoni. Un gran muro para contener la agresividad del mar (erosión continua y catástrofes a causa de las tempestades) sigue toda la costa de este tramo. El paseo a lo largo de este dique es una actividad muy comun. Entre las grandes piedras se encuentran una serie de construcciones tipo cabañas hechas a partir de los materiales que trae el mar durante las tempestades que se usan para fiestas y comidas durante el verano. En el centro de este muro se encuentra la localidad de Malamocco que tiene una población envejecida que participa activamente en el territorio (paseos en el Murazzi, huertos tanto públicos como privados, ocupación de las plazas, etc.). Un gran fuerte separa el pueblo del mar Adriático. Este fuerte contiene distintas edificaciones militares más recientes, siendo una superposición de estratos histórico-militares. El fuerte tiene un sistema de canales y fosas con el agua salobre de la laguna. Actualmente están colonizados por plantas de ambiente halófilo. El resto del territorio son grupos de vivienda colectiva o terceros paisajes ${ }^{3}$. La "naturalización" de estos espacios es el término que usa el plan regulador aprobado por las autoridades de la isla para la proyección de esta zona del Lido. Creemos que el marco histórico de este ámbito se basa en la humanización del paisaje, los buertos salados, asi que la propuesta de regeneración de esta zona tendría que continuar ligada a las actividades antrópicas y la laguna. La población envejecida pero activa de Malamocco sostiene el discurso ya que utiliza antiguas zonas agrícolas como actuales buertos públicos con la recuperación de antiguos canales de agua salobre de la laguna. El paseo entre el Murazzi y las zonas militares abandonadas densamente cubiertas con especies halófilas es una de las actividades más recurrentes. El Murazzi es el tramo más denso en edificios militares de todas las épocas (fuerte, baterías y bunkers). Estas construcciones tienen un nivel mucho más elevado y ofrecen unas vistas panorámicas imposibles de obtener desde ninguna otra construcción ya que el espacio donde se hallan erguidas es terreno no construible. Nuestra propuesta pretende equilibrar a través de una intervención las actividades locales como los huertos o la autoconstrucción en lugares públicos (Egg, 2010) con la continuidad de las grandes entidades vegetales y patrimoniales tan presentes en este tramo.
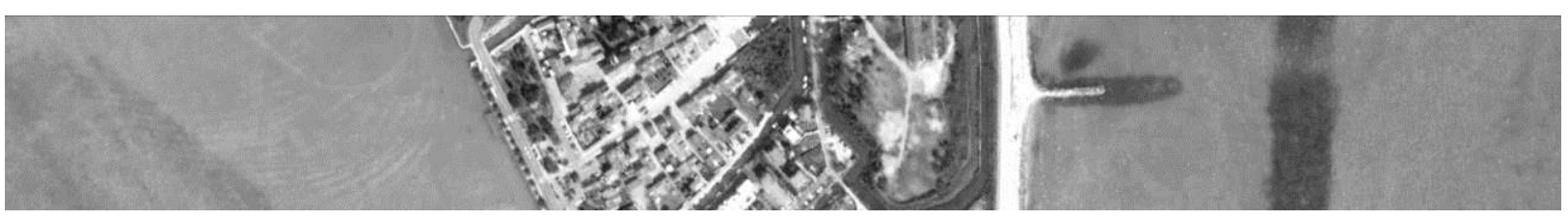

Fig. 12. Foto aérea del ámbito de Malamocco

- El ámbito de Punta Alberoni, en el sur, se caracteriza por la relativamente buena conservación del ecosistema litoral marítimo así como del ecosistema lagunar. Esta parte del territorio litoral está bajo la protección del plan europeo Natura 2000. Su gran fuerte fue destruido pero quedan las trazas de las murallas y fosas puntiagudas así como las fortificaciones, baterías y bunkers construidos posteriormente a lo largo de la entrada a la laguna desde el mar.

Recuperar la transición ecológica entre la laguna y el mar es el objetivo de la intervención en el punto más meridional de la isla. Este tramo es clave para entender las dinámicas naturales de la

\footnotetext{
3 Teoría desarrollada por Gilles Clément (2003): “The Third Landscape - an undetermined fragment of the Plantary Garden -designates the sum of the space left over by man to landscape evolution - to nature alone. Included in this category are left behind (délaissé) urban or rural sites, transitional spaces, neglected land (friches), swamps, moors, peat bogs, but also roadsides, shores, railroad embankments, etc.".
} 
isla litoral: es el único que contiene un número de entidades expectantes suficientemente importantes entre la laguna y el mar para poder rehacer la conexión ecológica.

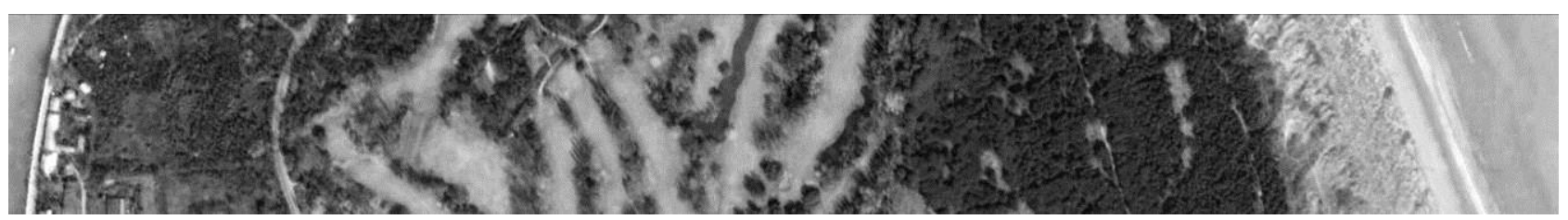

Fig. 13. Foto aérea del ámbito de Punta Alberoni

\section{Conclusión}

La propuesta sobre cómo regenerar la isla del Lido a través de la interpretación de sus paisajes culturales revela que la superposición y análisis de los estratos característicos de una región -un conjunto de características biofísicas, patrimoniales, geográficas, económicas y sociales específicas- expresan relaciones que pueden utilizarse como fundamentos para la intervención en el territorio. Estas relaciones se traducen en lugares físicos pero también en estrategias y dispositivos de proyecto. En el caso del Lido de Venecia, la interacción entre las comunidades vegetales y zonas históricas abandonadas nos ha inspirado un tipo de intervención a una escala global que identifica los elementos de coherencia del territorio de la laguna de Venécia, pero que se desarrolla diferentemente según la diversidad de contextos locales de la isla del Lido de Venecia.

\section{Referencias}

Clément, G. (2003): Manifeste du Tiers-Paysage. Editions Sujet/Objet. Paris

Egg, A.L. (2010): Kinya Maruyama. Ed. Actes du sud, Arles.

Fabian, L. (2010): Extremecity: Climate change and landspcae transformation of waterscape, IUAV, Venecia.

Galí-Izard, T. (2006): The same landscapes. Gustavo Gili, Barcelona.

McHarg, I.L. (2000): Proyectar con la naturaleza. Gustavo Gili, Barcelona.

Guerzoni, S.; Tagliapietra, D. (2006): Atlante della laguna. Marsilio Editori, Venecia.

Virilio, P. (1994): Bunker archéologie. Ed. Du Demi-Cercle, Paris. 